\title{
Anwerben allein reicht nicht
}

\section{Über den Einsatz ausländischer Fach- und Pflegekräfte in Deutschland}

\section{MARTIN BECK}

Prof. Martin Beck ist geschäftsführender Gesellschafter der Beck Management Center $\mathrm{GmbH}$ in Tübingen. Er begleitet die Sozialwirtschaft seit fast 30 Jahren aus verschiedenen Perspektiven. Er ist heute vor allem als Personalberater für Führungspositionen, als Aufsichtsrat in Sozialwirtschaft und Wirtschaft, als Hochschullehrer und Autor tätig.

www.prof-beck.net

\author{
Viele Sozialunternehmen, insbesondere aus der \\ Pflege, können nicht mehr genug Fachkräfte \\ auf dem deutschen Arbeitsmarkt finden. Doch \\ der Schritt zur Anwerbung im Ausland bedarf \\ sorgfältiger gesellschaftlicher, wirtschaftlicher \\ und nicht zuletzt ethischer Überlegungen.
}

Fachwelt und Medien sind sich einig: Es fehlt an qualifizierten Pflegekräften in Deutschland. Die veröffentlichten Zahlen schwanken derzeit von 3.000 bis 12.000 unbesetzten Stellen. Das ist in einem großen Land wie Deutschland noch keine Katastrophe, aber es könnte sich - mit regionalen Unterschieden noch zu einem schweren Problem ausweiten. Über den Zeitpunkt, zu dem ein kritischer Punkt erreicht sein wird, gibt es unterschiedliche Auffassungen. Auch bei anderen Berufsgruppen wie zum Beispiel Erzieherinnen oder Ärzten zeichnen sich ähnliche Problemstellungen ab.

Aus der Sicht der Führung eines Sozialunternehmens stellt sich die nüchterne Frage, ob die erforderliche Anzahl von Fachkräften mit der gewünschten Qualität im eigenen Land beschafft werden kann oder ob hierzulande deutliche Abstriche an Menge und Qualität gemacht werden müssen. Wenn wegen Personalmangels die Schließung von Wohnbereichen oder ganzen Einrichtungen droht, muss gehandelt werden. Eine leerstehende Einrichtung ist auf jeden Fall die schlechteste Variante. Auf Rang zwei der schlechten Varianten liegt die Senkung des fachlichen Niveaus, weil »halt keine guten Fachkräfte zu finden« seien.

Dieser Gedankengang führt schnell zu der Frage, wo außerhalb Deutschlands zu einem vertretbaren Aufwand und mit einem überschaubaren Risiko zusätzliche Fachkräfte gewonnen werden könnten. Das ist kein neuer Gedanke, er wurde aber bisher weitgehend individuell gedacht und kleinteilig um- gesetzt. Wer eben in einem Land jemand kannte, der Leute kannte, die vielleicht als soziale Fachkräfte nach Deutschland kommen könnten, der nutzte seine kleine Individualchance. Das wird in $\mathrm{Zu}$ kunft nicht mehr reichen.

\section{Wie ausländische Fachkräfte bezahlt werden sollten}

Es wird sicherlich die Versuchung geben, mit Hilfe der ausländischen Fachkräfte erst einmal Personalkosten einzusparen. Das ist ein naheliegender Gedanke, aber er sollte nicht ernsthaft weiter verfolgt werden. Sobald diese Fachkräfte eingearbeitet sind, werden sie ihren wahren Marktwert erkennen und dann entweder unzufrieden werden und Forderungen stellen - oder einfach gehen. Deshalb lautet die Empfehlung: Nicht die Personalkosten sollten im Vordergrund stehen, sondern die Qualifikation und Qualität.

\section{Welche zusätzlichen Aufwendungen zu erwarten sind}

Ja, es ist mit erheblichen Aufwendungen zu rechnen. Das gilt unabhängig davon, ob sich ein Unternehmen selber auf den spannenden Weg macht, irgendwo in Europa arbeitswillige Fachkräfte aufzuspüren und mit ihnen den langen Weg zur in Deutschland anerkannten Fachkraft zu gehen oder ob sich das Unternehmen eines Partners bedient. Bisher gibt es keine seriösen und übertragbaren Kostenrechnungen. Einige 
der Kostenarten können aber beispielhaft benannt werden:

- Honorare der lokalen Vermittlungsagenturen im Herkunftsland

- Honorare der Beratungs- oder Begleitungspartner in Deutschland

- Reisekosten für Bewerber nach Deutschland oder für Führungskräfte ins Herkunftsland der Bewerber

- Kosten der im Heimatland oder in Deutschland durchgeführten fachbezogenen Sprachkurse

- Kosten der Anerkennung der im Ausland erworbenen Qualifikationen

- Kosten der Unterbringung am Einsatzort (sofern nicht ortsübliche Mieten verlangt werden)

- soziale Betreuung in den ersten Arbeitsmonaten (Freizeitprogramm, Integrationsübungen, Alltagstraining)

- Kosten für Mentoring am Arbeitsplatz etc.

All diese Kosten sind nicht zu vernachlässigen. Wer hier aber an der falschen Stelle spart, erhöht das Risiko des Scheiterns. Scheitern bedeutet in diesem $\mathrm{Zu}$ sammenhang: Alles von vorne! Stelle unbesetzt, Kosten vergeblich ausgegeben, neuer Prozess mit neuen Risiken.

\section{Was man selber machen kann, was besser gemeinsam, was lieber mit Dienstleistern}

Das Grundproblem der Gewinnung und Betreuung ausländischer Fachund Pflegekräfte liegt darin, dass eine Vielzahl von Prozessen gleichzeitig aufgenommen, in Gang gehalten und zu einem Ergebnis geführt werden müssen, obwohl sie ungleichzeitig verlaufen und von unterschiedlichen Beteiligten mit unterschiedlichen Interesse begleitet oder behindert werden. Die jeweiligen Partner dieser Prozesse sind teilweise im Inland, teils im Ausland angesiedelt. Es ist immer mit Überraschungen zu rechnen. Wer rechnet zum Beispiel damit, dass sich die Anerkennung der Berufsqualifikation eines Menschen aus einem EU-Land unter EU-Recht durch deutsche Behörden oft Monate hinziehen kann und dann auch noch einen respektablen dreistelligen Eurobetrag kostet? Dass Deutschland diese Kräfte dringend braucht, scheint den Prozessbeteiligten nicht immer klar (oder klar gemacht worden) zu sein.

\section{Besonderheiten kirchennaher Organisationen}

Für die in Deutschland marktführenden kirchlichen Anbieter Diakonie und Caritas sind Pflegekräfte aus überwiegend muslimisch geprägten oder durch Jahrzehnte kommunistischen Regimes weitgehend religionsfrei gemachten Ländern ein Problem. Sie stehen unter verstärktem Druck ihrer Kirchen, überwiegend eigene Leute zu beschäftigen. Dieses hohe Ziel lässt sich nur mit regionalen Unterschieden verwirklichen. Bei der Gewinnung von ausländischen Fachkräften wirft dieses Ziel neue Fragen auf. Die romanischen Länder und Polen bieten überwiegend römisch-ka-
Diesen Aufwand wird die Sozialwirtschaft nicht treiben können oder wollen, aber die Frage nach dem persönlichen Umfeld der jeweiligen Fachkraft kann nicht verdrängt werden. Wer hier mit Heimweh und Einsamkeitsgefühlen sitzt, der wird nicht auf Dauer die volle emotionale und fachliche Leistung bringen können. Weil wir aber über mäßig bezahlte Berufstätigkeiten sprechen, ist der Familiennachzug, selbst wenn er aufenthaltsrechtlich möglich wäre, häufig keine Option.

Vereinfacht kann die Regel aufgestellt werden: Je weiter der Sprach- und Kulturraum entfernt ist, desto mehr Aufmerksamkeit muss der Kulturfra-
"Viele Prozesse zum Anwerben und zum Einsatz ausländischer Fachkräfte müssen gleichzeitig angestoßen werden« tholisches Personal. Griechenland, Zypern und einige Balkanländer verfügen über eine größere Zahl von orthodoxen Christen (was für beide Verbände akzeptabel ist). Der Balkan insgesamt und Osteuropa ist in den vergangenen Jahrzehnten großflächig entkirchlicht worden. Viele Arbeitsbewerber können auf die Religions- oder Konfessionsfrage keine klare Antwort geben. Das sind Spezialfragen, die aber die beiden marktführenden Verbände betreffen und deshalb nicht vernachlässigt werden können.

\section{Kulturelle Fragen}

Es ist zu vermuten, dass viele der potenziellen ausländischen Pflegekräfte zum ersten Mal länger und zum ersten Mal in einem beruflichen Zusammenhang im Ausland sein werden. Das wirft neben der Sprache insbesondere kulturelle Fragen auf.

International tätige Firmen bereiten Mitarbeiter, die in einem anderen Land tätig werden sollen, systematisch auf die dort vorfindliche und zu respektierende Kultur vor. Wo Familien mitziehen, werden auch diese unterstützt, und zwar vor der Ausreise, während des dortigen Aufenthalts und nach der Rückkehr. ge und dem entsprechenden Training gewidmet werden. Der Autor ist zum Beispiel mit Partnern in der Ostslowakei (wo die Ukraine deutlich näher liegt als Mitteleuropa) und in Albanien (das jahrzehntelang, fast wie Nordkorea, von der Außenwelt abgeschlossen war) im Kontakt. Beide Länder weisen hohe Arbeitslosigkeit und einen hohen Jugendanteil auf. Sie kämen also als Herkunftsländer infrage. Aber der unverzichtbare Integrations- und Begleitaufwand wird hoch sein.

\section{Fachliche Fragen}

Die Vorstellungen von Sozialer Arbeit und von Pflege variieren von Land $\mathrm{zu}$ Land. Andere Traditionen, andere Gesetzgebung, teilweise andere Ausbildungsgänge und Qualifikationsanforderungen sind anzutreffen. Vermutlich wird kein einziger Neuzugang aus einem anderen Land ohne eine gewisse - möglichst qualifiziert unterstützte Anpassungsleistung wirksam eingesetzt werden können.

Diese Fragen müssen offensiv und proaktiv aufgegriffen werden, bevor peinliche Missverständnisse oder gar grobe Fehler vorkommen. Wer diesen Punkt unterschätzt, vielleicht aus Sparsamkeitsgründen, geht ein hohes Risiko ein. $\quad \rightarrow$ 


\section{Thesen zur Standortbestimmung}

1 Bei der Anwerbung und dem Ein- satz ausländischer Fachkräfte wird es nicht ohne gezielte und ernsthafte Integrationshilfen gehen. Das kostet Zeit und Geld, ist aber für den Erfolg unverzichtbar.

2 Es gibt keine schnellen Erfolge, wenn _. man einmal von Zufallstreffern im einstelligen Bereich absieht.

3 Es ist-auch für große Unternehmen3.empfehlenswert, sich jeweils auf ein oder zwei Länder zu konzentrieren, um sowohl die Integrationsbemühungen zu erleichtern als auch die praktischen Prozesse zu vereinfachen.

1 Die beste Lösung wäre, mit langem - Atem in eine auf den deutschen Markt ausgerichtete (aber natürlich auch im Heimatland hoch anerkannte) Qualifikation zu investieren. Für die überwiegend kurzfristig agierenden Sozialunternehmen aller Größen wäre das aber ein großer Schritt, der vermutlich nur von mehreren starken Partnern gemeinsam gegangen werden könnte.

Martin Beck

\section{Fragen der \\ Organisationsentwicklung}

Mit ausländischen Fachkräften kommen ja nicht nur Probleme in die Häuser, sondern auch Potenziale. Die »Kundschaft« ist längst multikulturell, da kann es nur hilfreich sein, wenn auch das Personal aus verschiedenen Kulturkreisen und Sprachwelten kommt. Einfach ist das im Betrieb nicht, aber es ist heute in vielen Wirtschaftszweigen längst der Normalzustand. Die Sozialwirtschaft muss sich hier noch bewegen. Beschäftigte aus anderen Kulturen bringen andere Vorstellungen von Arbeit, von Führung, von Arbeitsabläufen mit. Auch diese müssen proaktiv bearbeitet werden.

\section{Ethische Fragen}

Ganz zu Recht wird im Zusammenhang unseres Themas die Frage gestellt, ob es denn ethisch korrekt sei, junge oder auch ältere Menschen aus ihrem heimatlichen, kulturellen und sprachlichen $\mathrm{Zu}$ sammenhang heraus zu lösen und nach Deutschland zu holen. Schließlich sei ja unser Problem nicht deren Problem.

Das ist in der Theorie korrekt. In der Praxis ist es allerdings so, dass unser Problem eher deren Chance ist. Wer als Jugendlicher in einer Gesellschaft ohne Arbeits- und Ausbildungschance lebt, wird nüchtern seine Möglichkeiten abschätzen. Wer als Erwachsener, sei er alleine oder mit Versorgungsverpflichtungen für Angehörige, eine Qualifizierungs- und Beschäftigungschance erhält, wird sie ergreifen.

Es besteht für uns kein Anlass, deshalb mit schlechtem Gewissen da zu stehen. Eher im Gegenteil. Das hat auch die Bundesregierung erkannt und leistet mit dem Programm »MobiPro-EU « zugunsten ausbildungswilliger junger Menschen bis 35 Jahren aus Europa einen interessanten Beitrag zum Thema.

\section{Probleme und Feststellungen}

Es gibt einige Aspekt, auf die in diesem Zusammenhang hingewiesen werden muss:

- Offenbar gelingt es in Deutschland bisher nicht in wünschenswertem Umfang, Migrantinnen und Migranten für Pflegeberufe zu gewinnen.

- Offenbar ist es - auch bei Fachkräften aus EU-Mitgliedsländern - bisher nicht möglich, ein zügiges und respektvolles Anerkennungsverfahren für die im Ausland erworbenen beruflichen Qualifikationen einzurichten.

- Die im Ausland erwerbbaren Sprachkenntnisse reichen häufig nicht aus, um im Pflegealltag die Zwischentöne $\mathrm{zu}$ verstehen und mit besorgten Angehörigen oder kommunikativ eingeschränkten Heimbewohnern adäquat $\mathrm{zu}$ kommunizieren. Eine intensive fachsprachliche Schulung, ausgerichtet am beruflichen Alltag, ist unabdingbar.

An diesem Punkt wird der Autor etwas zurückhaltend, weil er sich zu seiner eigenen Überraschung innerhalb weniger Monate vom fachlich-distanzierten Beobachter des Themas zum engagierten Treiber, zum Gesprächspartner in mehreren Entsendeländern und zum Seminarveranstalter entwickelt hat. Das war nicht der Plan, aber der Sog aus

\section{Dienstleister - ja oder nein?}

Deutschland ist so stark - teilweise auch der Druck aus Ländern mit miserabler Wirtschafts- und Soziallage -, dass aktives Handeln angezeigt war und ist.

Es gibt bisher keinen Anbieter auf dem Markt, der interessierten deutschen Unternehmen eine Full-Service-Dienstleistung anbieten kann. Auch weiß bisher niemand, was diese Dienstleistung, die ja über einen längeren Zeitraum und viele Hürden erbracht werden müsste, kosten würde.

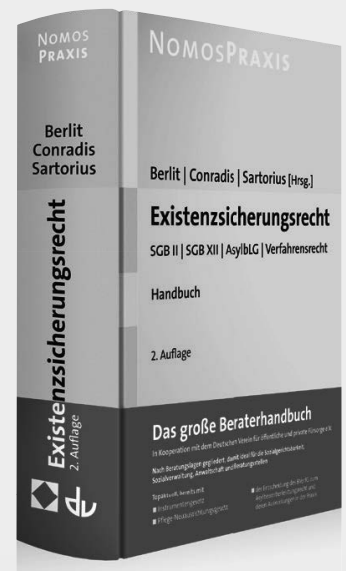

\section{Existenzsicherungsrecht} SGB II | SGB XII | AsylbLG | Verfahrensrecht

Handbuch

Herausgegeben von VRiBVerwG Prof. Dr. Uwe Berlit, RA Dr. Wolfgang Conradis, FASozR, und RA Dr. Ulrich Sartorius, FAArbR u FASOzR

2. Auflage 2013, 1.212 S., geb., 89,- €, ISBN 978-3-8329-4709-5

Dieses Handbuch sichert Ihre Existenz in der Beratungspraxis, sei es als Anwalt oder in einer Beratungsstelle: Von der Grundsicherung für Arbeitssuchende über die Sozialhilfe bis zu den Leistungen an Asylbewerber werden alle Fragen im Zusammenhang mit dem Recht der Existenzsicherung umfassend beantwortet.

www.nomos-shop.de/11523

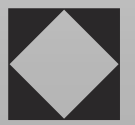

Nomos 